\title{
EVALUACIÓN DE MÉTODOS DE CONCENTRACIÓN Y PURIFICACIÓN DE Giardia spp. A PARTIR DE MUESTRAS COPROLOGICAS
}

\author{
Kathia Tarqui Terrones 1,2,a, Giovanna Ramírez Carranza 1,2,b, María Beltrán Fabián 1,2,c
}

\begin{abstract}
RESUMEN
El objetivo del estudio fue comparar diferentes métodos de concentración para recuperar la mayor cantidad de quistes de Giardia spp. a partir de muestras coprológicas. Se analizaron 100 muestras procedentes de hospitales de referencia nacional y se aplicaron cuatro métodos parasitológicos: concentración por sedimentación espontánea en tubo (TSET), Faust, gradiente de sucrosa de una fase y gradiente de sucrosa de dos fases. Se encontró que el método de gradiente de sucrosa de dos fases alcanzó resultados significativamente mejores en concentración de quistes (121 903 quistes $/ \mathrm{ml}$ ) y cantidad de detritos (6\%), en comparación con los métodos de Faust (35 355 quistes $/ \mathrm{ml}$ ), concentración por sedimentación espontánea en tubo $(20,145$ quistes $/ \mathrm{ml}$ ) y gradiente de sucrosa de una fase (18 702 quistes $/ \mathrm{ml})$. Se concluye que el método más eficaz para la concentración y purificación de quistes de Giardia spp. a partir de muestras coprológicas es el método de gradiente de sucrosa de dos fases, lo que facilitaría los cultivos in vitro de Giardia spp.
\end{abstract}

Palabras claves: Giardia spp.; Sacarosa; Gradiente de densidad; Sedimentación en tubo; Recuento de células (fuente: DeCS BIREME).

\section{EVALUATION OF METHODS OF CONCENTRATION AND PURIFICATION OF Giardia spp. FROM COPROLOGICAL SAMPLES}

\begin{abstract}
The aim of this study was to compare different methods of concentration to recover the largest number of Giardia spp. cysts from coprological samples. One hundred (100) samples from national reference hospitals were analyzed and four parasitological methods were applied: spontaneous tube sedimentation concentration (TSET), Faust, single-phase sucrose gradient, and two-phase sucrose gradient. The two-phase sucrose gradient method was found to achieve significantly better results in cyst concentration (121,903 cysts $/ \mathrm{ml}$ ) and amount of debris $(6 \%)$, compared to Faust methods $(35,355 \mathrm{cysts} / \mathrm{ml})$, spontaneous tube sedimentation concentration $(20,145 \mathrm{cysts} / \mathrm{ml})$, and single-phase sucrose gradient $(18,702 \mathrm{cysts} / \mathrm{ml})$. It is concluded that the most effective method for the concentration and purification of Giardia spp. cysts from coprological samples is the two-phase sucrose gradient method, which would facilitate in vitro culture of Giardia spp.
\end{abstract}

Keywords: Giardia spp.; Sucrose; Density gradient; Tube sedimentation; Cell count (fuente: DeCS BIREME).

\section{INTRODUCCIÓN}

En el mundo, la giardiasis es una de las principales infecciones gastrointestinales de impacto en salud pública (1). En Asia, África y América Latina, alrededor de 200 millones de personas padecen esta enfermedad con aproximadamente 500000 nuevos casos reportados cada año ${ }^{(2)}$. En Perú, la prevalencia de giardiasis se encuentra alrededor de $15 \%$ a $18 \%$, con cifras que varían desde $17,8 \%$ en la costa, $15,4 \%$ en la sierra y $5 \%$ en la Selva ${ }^{(3)}$.

Giardia spp. es un parásito protozoario flagelado de los seres humanos y otros mamíferos, habita en el intestino delgado del hospedero, su infección puede ocasionar malnutrición y retraso del crecimiento ${ }^{(4)}$. Su transmisión se da por la ingesta de alimentos o agua contaminada con formas evolutivas del parásito. Presenta dos estadios

\footnotetext{
Laboratorio de Referencia Nacional de Enteroparásitos, Instituto Nacional de Salud. Lima, Perú

Facultad de Ciencias Biológicas, Universidad Nacional Mayor de San Marcos. Lima, Perú

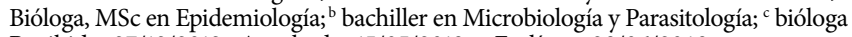

Recibido: 27/12/2018 Aprobado: 15/05/2019 En línea: 28/06/2019
}

Citar como: Tarqui Terrones K, Ramírez Carranza G, Beltrán Fabián M. Evaluación de métodos de concentración y purificación de Giardia spp. a partir de muestras coprológicas. Rev Peru Med Exp Salud Publica. 2019;36(2):275-80. doi: http://dx.doi.org/10.17843/rpmesp.2019.362.4151. 
evolutivos claramente diferenciados que se adaptan a distintas condiciones ambientales: el trofozoito, la forma vegetativa que es responsable de los síntomas de la enfermedad; y el quiste, la forma de resistencia que es responsable de la transmisión de huésped a huésped ${ }^{(5)}$ y principal forma diagnóstica del parásito. El quiste tiene forma oval con un tamaño promedio de 6-10 $\mu \mathrm{m}$, pudiendo llegar a observarse de dos a cuatro núcleos ${ }^{(6)}$.

Los métodos coproparasitológicos de concentración son muy útiles para la detección de parásitos; sin embargo, para estudios de cultivo in vitro y ensayos de susceptibilidad antiparasitaria se necesita que los quistes puedan recuperarse en mayor cantidad, con el menor número de detritos y sin alteraciones morfológicas (7). Los métodos de concentración descritos en la literatura se basan en gradientes de densidad o en soluciones con sacarosa ${ }^{(8,9)}$. Asimismo, se han reportado metodologías basadas en el uso de percoll (sílice coloidal recubierta con polivinilpirrolidona); sin embargo, la osmolaridad de los reactivos puede afectar negativamente la viabilidad del quiste ${ }^{(10,11)}$.

Este parásito puede llegar a cultivarse, por esta razón, la exquistación in vitro es una alternativa que abre horizontes para el desarrollo de pruebas de susceptibilidad antiparasitaria. Sin embargo, uno de los principales problemas es aislar los quistes presentes en las muestras coprológicas y separarlos de los detritos u organismos entéricos.

En el Perú, son escasos los trabajos asociados al cultivo de enteroparásitos, lo que motivó a buscar metodologías efectivas que permitan concentrar parásitos intestinales con poca contaminación, a fin de implementar cultivos axénicos y ensayos de susceptibilidad antiparasitaria. El objetivo del estudio fue comparar métodos de concentración que permitan recuperar la mayor cantidad quistes de Giardia spp. de forma rápida y efectiva, a partir de muestras coprológicas y con menor cantidad de detritos.

\section{EL ESTUDIO}

\section{DISEÑO Y MARCO MUESTRAL}

Se realizó un estudio observacional descriptivo. Se trabajó con 100 muestras fecales humanas positivas a Giardia spp. procedentes de tres hospitales de referencia nacional de Lima (Hospital Arzobispo Loayza, Hospital Cayetano Heredia y Hospital Sergio Bernales), obtenidas por muestreo no probabilístico intencionado durante mayo de 2016 a diciembre de 2017. Se incluyeron muestras que sólo presenten el parásito Giardia spp. en una carga parasitaria promedio de cinco quistes por campo microscópico, sin contener ningún fijador parasitológico.

\section{MUESTRAS ANALIZADAS}

Se analizaron 100 muestras coprológicas que fueron transportadas en un cooler y conservadas a $4{ }^{\circ} \mathrm{C}$ por un

\section{MENSAJES CLAVE}

Motivación para realizar el estudio. En los últimos años se ha incrementado la resistencia a las drogas antigiardiásicas. Se ha realizado un estudio que compara diversos métodos de concentración de quistes de Giardia spp. a partir de muestras coprológicas.

Principales hallazgos. Los resultados indican que el método de gradiente de sucrosa de dos fases fue el más eficaz para la concentración de quistes, con poca cantidad de detritos, en comparación con los métodos de gradiente de sucrosa de una fase, Faust y de concentración por sedimentación espontánea en tubo.

Implicancias. Este estudio es la base para realizar investigaciones sobre resistencia antiparasitaria in vitro.

periodo máximo de 24 horas después de su obtención. Luego fueron confirmadas por microscopía en el Laboratorio de Referencia Nacional de Enteroparásitos, mediante examen parasitológico directo.

Los criterios empleados para evaluar los métodos de concentración fueron: mayor recuperación de quistes por mililitro, menor cantidad de detritos y no presentar cambios en la morfología del quiste.

\section{MÉTODOS EVALUADOS}

Las muestras coprológicas (aproximadamente $2 \mathrm{~g}$ ) se homogenizaron con solución salina y se evaluaron para los siguientes métodos:

Técnica de concentración por sedimentación espontanea en tubo (TSET)

Este método se basa en el uso de agua $u$ otros líquidos de baja densidad (en este caso, solución salina fisiológica), recuperando las formas microscópicas evolutivas de los parásitos en el sedimento que se forma en el fondo del tubo, donde se depositan por su densidad. En este método es posible detectar quistes y trofozoitos de protozoos ${ }^{(12)}$.

\section{Método Faust}

Este método se basa en la propiedad que tiene la solución de mayor densidad para hacer flotar elementos menos densos. Se usa una solución de sulfato de zinc, cuya densidad específica es 1,180 (33\%) la cual es más alta que la de la mayoría de los quistes, los residuos se mantienen en el fondo del tubo. La concentración de parásitos por flotación permite verificar la existencia de quistes de protozoos incluso cuando están presentes en pequeñas cantidades; el sobrenadante recuperado se centrifugó a $2300 \mathrm{rpm}$ y se realizaron tres lavados con agua destilada para eliminar el sulfato de zinc ${ }^{(13)}$.

\section{Gradiente de sucrosa de una fase}

Este método se basa en la separación de las partículas según su densidad de flotación ${ }^{(8)}$. Se añadieron $9 \mathrm{ml}$ de 
solución salina al sedimento y se centrifugaron durante 5 minutos a $1500 \mathrm{rpm}$. Luego se agregaron $4 \mathrm{ml}$ de PBS (Phosphate-buffered saline) y $4 \mathrm{ml}$ de solución de sucrosa (densidad 1,275) a través de las paredes. Se centrifugó a 2300 rpm durante 10 minutos. La fase de sucrosasedimento se aspiró con una pipeta de transferencia, luego se centrifugó y se realizaron tres lavados con agua destilada para eliminar la sucrosa.

\section{Gradiente de sucrosa de dos fases}

Este método también se basa en la separación de elementos según su densidad de flotación y se utilizan dos soluciones de diferentes densidades de azúcar (14,15). El sedimento se mezcló volumen / volumen con solución de sucrosa 0,85 M, luego se centrifugó a 2300 rpm durante 10 minutos a $4{ }^{\circ} \mathrm{C}$, se recuperó la fase sucrosa-agua y se realizaron 3 lavados con agua destilada. Posteriormente, se preparó un tubo con gradiente de $0,85 \mathrm{M}$ a 0,4 $\mathrm{M}$ y se agregó cuidadosamente el sedimento en la parte superior, se centrifugó a $2300 \mathrm{rpm}$ durante 10 minutos a $4^{\circ} \mathrm{C}$ y se recolectó la interfase. Luego se centrifugó a $2300 \mathrm{rpm}$ a $4{ }^{\circ} \mathrm{C}$ durante 5 minutos para eliminar la sucrosa.

\section{PROCEDIMIENTOS}

La estimación de la carga parasitaria obtenida de cada método se realizó a través del recuento de quistes por mililitro, se empleó un volumen conocido de muestra $(30 \mu \mathrm{l})$ y la fórmula que se utilizó fue la siguiente:

$N^{\circ}$ de quistes de Giardia spp $(q / m l)=\frac{\text { (Número de quistes contados) } \times 10^{3}}{\text { factor volumen } \times \text { factor de dilución }}$
Factor volumen= volumen analizado de la muestra en microlitros $(\mu \mathrm{l})$

Asimismo, se evaluó si las soluciones empleadas en los métodos de concentración inducían alguna alteración en la morfología del parásito, cambios en la forma oval o redondeada del quiste de Giardia spp.; adicionalmente se evaluó la cantidad de detritos observados en las muestras (escasos, moderados o abundantes) luego de la aplicación de los métodos en la lectura microscópica.

\section{ÁNALISIS DE DATOS}

Se analizó el rendimiento de cada método, a través de la comparación de medias obtenidas en los recuentos de quistes por mililitro $(q / \mathrm{ml})$. Se aplicó la prueba de análisis de varianza (ANOVA) de una vía, a fin de determinar si existían diferencias significativas; asimismo, se realizó la comparación múltiple de medias con la prueba post hoc de HSD Tukey. En el caso de las variables cualitativas (morfología del quiste y cantidad de detritos) se expresaron como porcentajes. Se aceptó un valor de $p<0,05$ como estadísticamente significativo. Se utilizó el programa estadístico SPSS versión 23 para Windows.

\section{HALLAZGOS}

Se realizó la lectura de las 100 muestras según cada método estudiado; fue evaluado por triplicado mediante examen parasitológico directo. Las medias de recuento por muestra se detallan en el Anexo1.

El método de concentración por sedimentación espontánea en tubo concentró, en promedio, 20145 quistes/ml, pero tuvo la limitación de presentar demasiados detritos (Figura 1a). El método de Faust concentró, en promedio, 35355 quistes/ml con escaso detritos (8\%); sin embargo, el sulfato de zinc alteró la morfología de la pared quística de Giardia spp. en mayor proporción (Figura 1b).

Al evaluar los métodos basados en soluciones de sucrosa, encontramos que la solución con densidad $1,275 \mathrm{~g} / \mathrm{ml}$, correspondiente al método de gradiente de sucrosa de una fase concentró, en promedio, 18702 quistes $/ \mathrm{ml}$ y presentó $94 \%$ de muestras con moderado a abundante detrito (Figura 1c). El método de gradiente de sucrosa de dos fases logró concentrar, en promedio, 121903 quistes/ml y presentó sólo 6\% de detritos (Figura 1d). En relación a las alteraciones morfológicas de los quistes según microscopia, se observó que el método Faust presentó un mayor porcentaje de deformidad de la pared quística (93\%), comparado con los otros métodos evaluados, sugiriendo su inviabilidad para cultivo (Tabla 1).

Según las pruebas de ANOVA y de Tukey existe diferencia significativa entre los métodos evaluados ( $p<0,01$ ), siendo el método de gradiente de sucrosa de dos fases el más eficaz en comparación a los otros métodos (Tabla 2, Figura 2).

Tabla 1. Evaluación entre cantidad de detrito y morfología de quistes en la muestras analizadas, según método evaluado

\begin{tabular}{lcccccc}
\hline \multirow{2}{*}{ Métodos } & \multicolumn{2}{c}{ Cantidad de detritos } & & \multicolumn{2}{c}{ Morfología del quiste } \\
\cline { 2 - 3 } \cline { 5 - 6 } & & Escaso (\%) & Moderado/Abundante (\%) & & Deformidad (\%) & Normal (\%) \\
\hline TSET $(n=100)$ & 0 & 100 & 8 & 5 & 93 & 7 \\
Faust $(n=100)$ & 92 & 94 & 6 & 6 & 94 \\
Sucrosa de una fase $(n=100)$ & 94 & 6 & & 6 & 94 \\
Sucrosa de dos fases $(n=100)$ & & & &
\end{tabular}

TSET: Técnica de concentración por sedimentación espontanea en tubo 


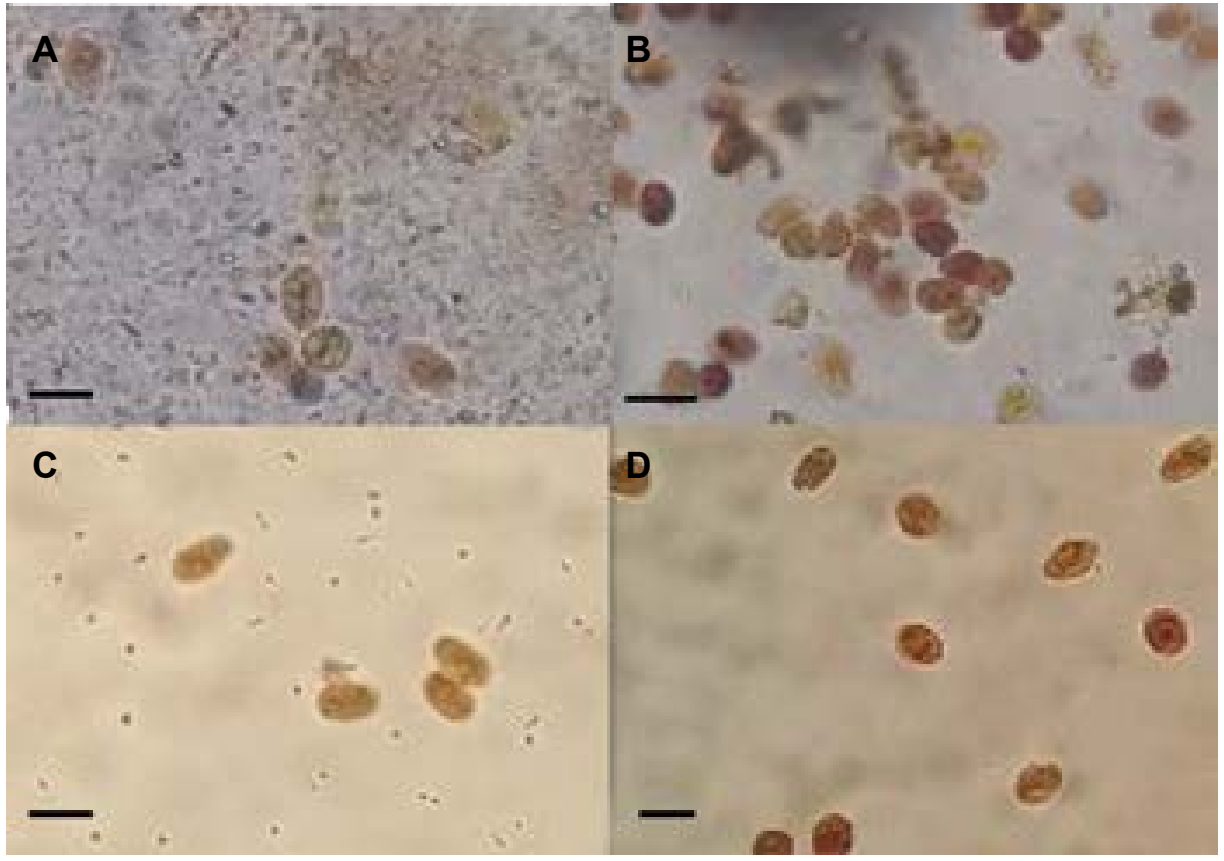

Figura 1. Visualización microscópica de quistes de Giardia spp. a 400X posterior a la aplicación de métodos de concentración. a) Los quistes después de la purificación con el Método Faust. b) Los quistes después de la purificación con sedimentación espontánea en tubo. c) Los quistes después de la purificación por el método de gradiente de sucrosa de una fase. d) Los quistes después de la purificación con una gradiente de sucrosa de dos fases. La barra indica $10 \mu \mathrm{m}$.

\section{DISCUSIÓN}

Los resultados demuestran que el método de concentración basado en gradiente de sucrosa de dos fases $(0,4 \mathrm{M}$ y $0,8 \mathrm{M})$ permite extraer una mayor cantidad de quistes de Giardia spp. a partir de muestras coprológicas, en comparación con los otros métodos evaluados $(p<0,001)$. La ventaja de este método es evidente y se refrenda con los reportes de Al -Tukhi el al. (14), Hautus et al. (15) y Visvesvara (16), quienes emplean este método para ensayos de cultivo in vitro, pero no especifican la carga parasitaria obtenida por muestra. Asimismo, cuando trabajamos con el método de sucrosa de una sola fase, contrariamente a lo reportado por otros autores ${ }^{(8,9)}$, se observó que la recuperación de quistes era baja y la presencia de detritos era de moderada a elevada en el $94 \%$ de las muestras. Además, se mostró las limitaciones del método de concentración basado en sedimentación simple, tanto en recuperación de quistes, como en presencia de abundantes detritos, motivo por el que se recomienda sólo con fines diagnósticos ${ }^{(12)}$.

Tabla 2. Comparaciones múltiples según métodos evaluados de acuerdo al recuento de quistes

\begin{tabular}{|c|c|c|c|c|}
\hline \multirow{2}{*}{ Métodos } & & \multicolumn{2}{|c|}{ Recuento de Giardia spp. (quistes/ml) } & \multirow{2}{*}{ Valor de $\mathrm{p}^{*}$} \\
\hline & & Media & $\mathrm{DE}$ & \\
\hline \multirow[t]{3}{*}{ TSET } & Faust & 35354 & 4663 & 0,556 \\
\hline & Sucrosa de una fase & 18701 & 1795 & 0,999 \\
\hline & Sucrosa de dos fases & 121903 & 15440 & $<0,001$ \\
\hline \multirow[t]{3}{*}{ Faust } & TSET & 20145 & 2311 & 0,556 \\
\hline & Sucrosa de una fase & 18701 & 1795 & 0,477 \\
\hline & Sucrosa de dos fases & 121903 & 15440 & $<0,001$ \\
\hline \multirow[t]{3}{*}{ Sucrosa de una fase } & TSET & 20145 & 2311 & 0,999 \\
\hline & Faust & 35354 & 4663 & 0,477 \\
\hline & Sucrosa de dos fases & 121903 & 15440 & $<0,001$ \\
\hline \multirow[t]{3}{*}{ Sucrosa de dos fases } & TSET & 20145 & 2311 & $<0,001$ \\
\hline & Faust & 35354 & 4663 & $<0,001$ \\
\hline & Sucrosa de una fase & 18701 & 1795 & $<0,001$ \\
\hline
\end{tabular}

TSET: Técnica de concentración por sedimentación espontanea en tubo, DE: Desviación estándar

*Prueba de HSD Tukey 


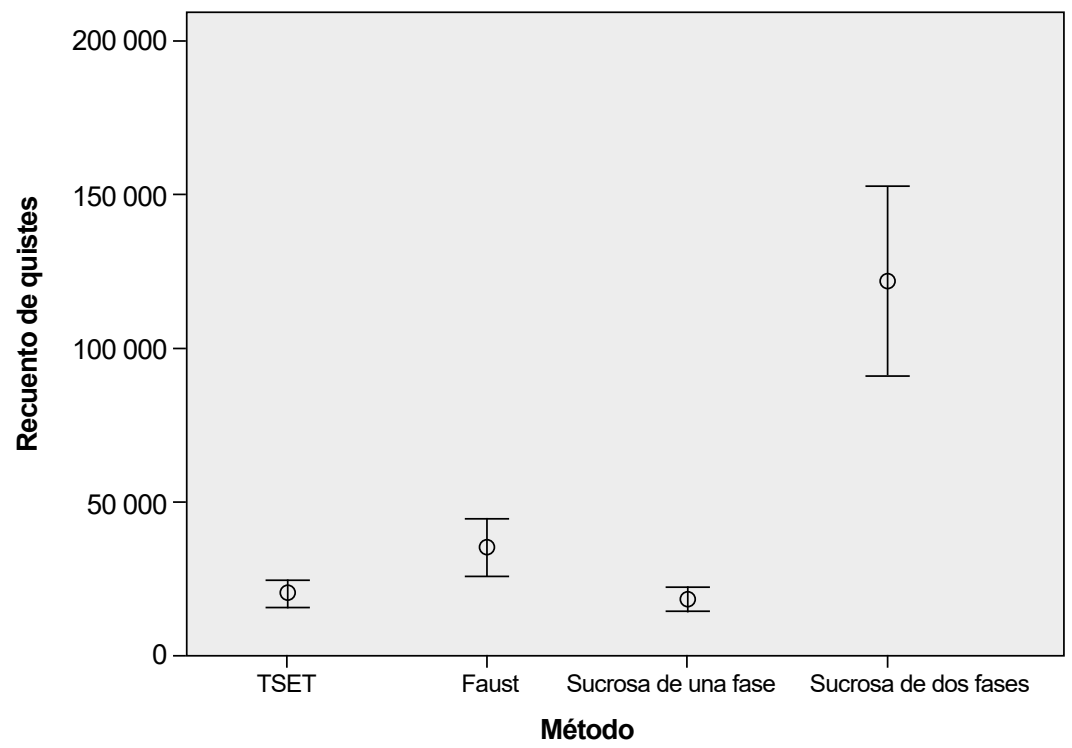

Barras de error: Intervalo de confianza de $95 \%$

TSET: Técnica de concentración por sedimentación espontanea en tubo

Figura 2. Recuento promedio de quistes de Giardia spp. según método evaluado

En relación al uso de reactivos empleados para quistes libres de contaminantes, nuestros resultados fueron similares a los reportados por otros autores ${ }^{(17,18)}$, quienes indican que químicos como el sulfato de zinc afectan las propiedades físicas y biológicas de los quistes, esto fue corroborado al evaluar el método Faust, que ocasionó deformación de la pared quística en $93 \%$ de las muestras, lo que dificultaría los posteriores procesos de exquistación del parásito in vitro, situación que no ocurre al emplear cloruro de sodio o sucrosa, donde se reporta una ligera deformidad en $5 \%$ a $6 \%$ de las muestras, respectivamente. Otros autores recomiendan la remoción de contaminantes por filtración ${ }^{(19)}$; sin embargo, se considera un método no muy práctico. De igual manera Barazesh et al. ${ }^{(7)}$ y Duque et al. ${ }^{(20)}$ usaron la solución de Percoll, para purificar quistes, no obstante, resulta ser un reactivo caro y causal de deformaciones de la pared quística.

Una de las limitaciones del estudio fue no determinar la viabilidad de los quistes recuperados por cada método, no permitiendo valorar la existencia de una relación significativa entre la alteración de la morfología del quiste y los patrones de exquistación de los mismos. Sin embargo, consideramos que los resultados de este estudio son importantes, ya que evidencian que es posible separar a los quistes parasitarios de los detritos fecales sin necesidad de técnicas muy complejas.

Se concluye que el método más eficaz para la concentración y purificación de quistes de Giardia spp. a partir de muestras coprológicas es el método de gradiente de sucrosa de dos fases, siendo un método sencillo y barato que podría emplearse como paso previo a los ensayos de cultivo y susceptibilidad parasitaria in vitro.

Agradecimientos: Agradecemos a los laboratorios de parasitología de los hospitales nacionales por el apoyo en la realización del presente proyecto.

Contribuciones de los autores: KTT participó en la concepción, diseño del artículo, análisis e interpretación de resultados. GRC realizó los experimentos y la base de datos. MBF participó en la interpretación de los resultados. Todos participaron en la redacción del artículo y aprobaron la versión final.

Fuentes de financiamiento: La presente investigación fue financiada con recursos de Fondo Nacional de Ciencia y tecnología (FONDECYT), Convenio N 136-2015.

Conflictos de interés: Los autores declaran no tener conflicto de intereses en la realización de este trabajo.

Material suplementario: Disponible en la versión electrónica de la RPMESP.

\section{REFERENCIAS BIBLIOGRÁFICAS}

1. Vásquez O, Campos T. Giardiasis. La parasitosis más frecuente a nivel mundial. Rev. del Centro de Inv. (Méx.). 2009;8(31):75-90.

2. Thompson A. The zoonotic significance and molecular epidemiology of Giardia and giardiasis. Vet Parasitol. 2004;126:1535. doi:10.1016/j.vetpar.2004.09.008.
3. Martinez E, Cerpa L, Liu M. Prevalencia de giardiasis en guarderías infantiles de Tiabaya-Arequipa, Perú 2006. Neotrop Helminthol. 2011;5(2): 257-64.

4. Berkman DS, Lescano AG, Gilman RH, Lopez SL, Black MM. Effects of stunting, diarrhoeal disease, and parasitic infection during infancy on cognition in late childhood: a follow-up study. Lancet. 2002; 359(9306):564-71. doi: 10.1016/S0140-6736(02)07744-9.

5. Adam RD. Biology of Giardia lamblia. Clin Microbiol Rev. 2001;14(3):447-75. doi: 10.1128/CMR.14.3.447-475.2001.

6. Midlej V, Benchimol M. Giardia lamblia behavior during encystment: 
how morphological changes in shape occur. Parasitol Int. 2009; 58(1): 72-80. doi: 10.1016/j.parint.2008.11.002.

7. Barazesh A, Majidi J, Fallah E, Gholikhani R. Introducing a simple and economical method to purify Giardia lamblia cysts. Afr J Biotechnol.2011;10(42): 84988501. doi: $10.5897 /$ AJB11.391.

8. Polverino D, Molina N, Minvielle M, Lozano E, Basualdo J. Técnicas de purificación y ruptura de quistes de Giardia spp. Rev argent Microbiol. 2006; 36(7):97-100.

9. Molina N. Epidemiologia molecular de Giardia lamblia en comunidades urbanas y rurales de Buenos Aires y Mendoza, Argentina [ Tesis de Magister]. Buenos aires: Facultad de Ciencias Médicas, Universidad Nacional de La Plata; 2009.

10. Sauch J. Purification of Giardia muris Cysts by Velocity Sedimentation. Appl Environ Microbiol. 1984;(48) 2:454-5.

11. Sauch J. Improved primary isolation for Giardia cysts. New Orleans: 83rd Annual Meeting of the American Society for Microbiolog; 1983.

12. Terashima A, Marcos L, Maco V, Canales M, Samalvides F, Tello R. Técnica de sedimentación en tubo de alta sensibilidad para el diagnóstico de parásitos intestinales. Rev Gastroenterol Perú. 2009;29 (4):305-310.

13. Beltrán M, Otárola J, Tarqui K. Manual de Procedimientos de laboratorio para el diagnóstico de los parásitos intestinales del hombre. Serie de normas técnicas $\mathrm{N}^{\circ} 37$. 2da Edición. Lima: Instituto Nacional de Salud; 2014. Disponible en: http://bvs. minsa.gob.pe/local/INS/165_NT37.pdf

14. Al-Tukhi MH, Al-Ahdal MN, Peters WA. simple method for excystation of Giardia lamblia cysts. Ann Trop Med Parasitol, 1991; 85(4), 427-31. doi: 10.1080/00034983.1991.11812587.

15. Hautus MA, Kortbee LM, Vetter JC, Laarman JJ. In vitro excystation and subsequent axenic growth of Giardia lamblia. Trans R Soc Trop Med Hyg. 1988; 82: 858-861. doi: 10.1016/00359203(88)90019-3.

16. Visvesvara G. Axenic growth of Giardia lamblia in Diamond's TPS1 medium. Trans R Soc Trop Med Hyg. 1980;74(2):213-215. doi: 10.1016/0035-9203(80)90249-7.

17. Blagg W, Schlogel EL, Mansour N. A new concentration technique for the demonstration of protozoa and helminth eggs in feces. Am J Trop Med Hyg.1955;4(1): 23-28. doi: 10.4269/ ajtmh.1955.4.23.
18. Walderich B, Mueller L, Bracha R, Knobloch J, Burchard G. A new method for isolation and differentiation of native Entamoeba histolytica and E. dispar cysts from fecal samples. Parasitol. Res.1997.83: 719-721. doi:10.1007/ s004360050.

19. Alvarado M., Wasserman M. Quick and efficient purification of Giardia intestinalis cysts from fecal samples Parasitol Res. 2006; 99: 300-2. doi: 10.1007/s00436-006-0143-x.

20. Duque-Beltran S, Nicholas-Orejuela R, Arévalo-Jamaica A, Guerrero-Lozano R, Montenegro S, James M. Detection of Giardia duodenalis antigen in human fecal eluates by enzyme-linked immunosorbent assay using polyclonal antibodies. Mem Inst Oswaldo Cruz. 2002; 97(8):1165-8. doi: 10.1590/ S0074-02762002000800018.

Correspondencia: Kathia Mariela Tarqui Terrones.

Dirección: Jr. Capac Yupanqui 1400 Jesús

María. Lima, Perú.

Teléfono: (+51) 997033653

Correoelectrónico:kathiax@yahoo.com 